\title{
Institutionelle Änderungen des Bundesrates
}

- Ein Beitrag zur Diskussion über eine Reform des föderalen Organs -

\section{Einführung}

Die historische Entscheidung für das Bundesstaatsprinzip im Grundgesetz schließt eine fortwährende Entwicklung und Anpassung der staatlichen Organisation an sich verändernde Rahmenbedingungen ein. Es sind vornehmliche Aufgaben der Politik, die Modernisierung der bundesstaatlichen Ordnung durch eine Reform des föderalen Staatsaufbaus Deutschlands voranzutreiben und der Verfassungslehre als „,wissenschaftliche

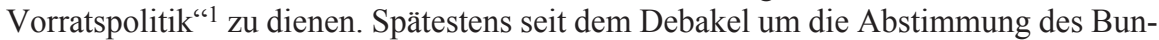
desrates über das Zuwanderungsgesetz am 22. März $2002^{2}$ sind die wiederholt behauptete parteipolitische Instrumentalisierung des Bundesrates als Organ zur Beteiligung der Länder an der bundespolitischen Willensbildung und die damit einhergehende Beeinträchtigung der beteiligungsföderalen Funktionsfähigkeit zu einem vielfach diskutierten Streitthema in der deutschen Verfassungsrechtslehre avanciert. ${ }^{3}$ Zeugnis dieses Streites sind auch die Diskussionen um die im November 2008 im Zusammenhang mit dem BKA-Gesetz wiederholt vorgeschlagene Änderung der Abstimmungsregel im Bundesrat. ${ }^{4}$

Die seit September 2006 in Kraft getretene erste Stufe der Föderalismusreform, ${ }^{5}$ die vor allem eine Reduzierung der Zustimmungsgesetze und mehr Kompetenzen für die Länder beinhaltet, ist eine Änderung der Entscheidungsfindung im Bundesrat schuldig geblieben. ${ }^{6} \mathrm{Da} \mathrm{zu}$ den erklärten Zielen der Reform die Verbesserung der Handlungsund Entscheidungsfreiheit von Bund und Ländern, die deutlichere Zuordnung der politischen Verantwortlichkeiten sowie die Steigerung der Effizienz und Zweckmäßigkeit bei der Aufgabenerfüllung zählen, wären insbesondere auch institutionelle Änderungen des Bundesrates als Organ zur Vertretung von Länderinteressen, „[...] in dem das fö-

1 Peter Häberle, Das Mehrheitsprinzip als Strukturelement der freiheitlich-demokratischen Grundordnung, in: JZ, 32 (1977) 8, S. 241.

2 Stenographischer Bericht BR, 774. Sitzung vom 22.3.2002, in: BR-Drs. 157/02, S. 131 ff., Abstimmung S. $171 \mathrm{ff}$.

3 Vgl. Gerrit Mulert, Der Bundesrat im Lichte der Föderalismusreform, in: DÖV, 60 (2007) 1, S. 26, m.w.N. in Fn. 7.

4 Schäuble will kleinen Parteien Einfluss nehmen, in: Süddeutsche Zeitung vom 22./23.11.2008, S. 1.

5 BGBl. 2006 I, 2034. Vgl. hierzu Peter M. Huber, Die Föderalismusreform I - Versuch einer Bewertung, in: Wolfgang Durner/Franz-Joseph Peine (Hrsg.), Reform an Haupt und Gliedern. Verfassungsreform in Deutschland und Europa, München 2009, S. 25 ff.

6 Vgl. Roland Sturm, Eine Bilanz aus politikwissenschaftlicher Sicht, in: Michael Borchard/Udo Margedant (Hrsg.), Der deutsche Föderalismus im Reformprozess, Sankt Augustin 2006, S. 192. 
deralistische Prinzip seinen organisatorischen Ausdruck finden sollte [... $]^{\text {“, }}$, zu erwarten gewesen. In Anbetracht der immer wiederkehrenden Diskussionen um institutionelle Fragen des Bundesrates, die vielfach als Grund für Probleme des Föderalismus angesehen werden, ${ }^{8}$ kann dies kein zufriedenstellendes Ergebnis darstellen. Auf der Grundlage normativer Bestandsaufnahmen und geltender Verfassungsprinzipien sowie unter Berücksichtigung von Reformvorschlägen soll nachfolgend hinterfragt werden, ob bestehende und vielfach kritisierte Funktions- und Effizienzdefizite des Bundesrates durch Änderungen in seiner Organisationsstruktur und/oder Verfahrens- bzw. Abstimmungsweise neutralisiert werden könnten.

\section{Zusammensetzung des Bundesrates}

\section{Bundesrats- oder Senatsmodell}

Im Gegensatz zu den Prinzipien von Demokratie und Rechtsstaat ist das Bundesstaatsprinzip keine ,unverzichtbare Regelausstattung moderner westlicher Verfassungsstaatlichkeit" , 9 so dass es hierzu andere legitime Alternativen gibt. ${ }^{10}$ In Deutschland ist die föderale Ordnung das Ergebnis eines historischen Prozesses (Verfassungsgeschichte), der sich vom Heiligen Römischen Reich über den Deutschen Bund und die Weimarer Republik bis hin zur (vereinten) Bundesrepublik ${ }^{11}$ zieht. Einen von der Verfassung vorgegebenen Begriff des Bundesstaatsprinzips sowie eine nach der Verfassung notwendige Anzahl von Gliedstaaten gibt es indes nicht. ${ }^{12}$ Deshalb ist auf seine Ausformung durch die Verfassung zurückzugreifen (Verfassungstheorie). Die Mitwirkung der Gliedstaaten an der Willensbildung des Bundes durch ein föderales Organ kann dabei unterschiedlich ausgeprägt sein. ${ }^{13}$

Es ist wiederholt hinterfragt worden, ob mit einer Änderung des Modells die Einflussnahme der politischen Parteien auf die Willensbildung im Bundesrat als einem föderalen Organ abgeschwächt werden könnte. Vorschläge zur Zusammensetzung nach dem Senatsmodell mit einer Kammer, bestehend aus unabhängigen von den Landtagen gewählten Einzelpersonen, hat es in der deutschen Verfassungsgeschichte mehrfach

7 Gerhard Leibholz/Dieter Hesselberger, Bundesrat und Parteienstaat, in: Bundesrat (Hrsg.), Der Bundesrat als Verfassungsorgan und politische Kraft, Bad Honnef/Darmstadt 1974, S. 102.

8 Vgl. Norbert Grube, Unverzichtbares Korrektiv oder ineffektive Reformbremse? Wahrnehmungen föderaler Strukturen und Institutionen in Deutschland, in: Jahrbuch des Föderalismus 5 (2004), S. $172 \mathrm{f}$.

9 Matthias Jestaedt, Bundesstaat als Verfassungsprinzip, in: Josef Isensee/Paul Kirchhof (Hrsg.), HdbStR, Band II, 3. Auflage, Heidelberg 2004, § 29, Rdnr. 1, S. 786.

10 Vgl. Ingo von Münch, Staatsrecht I, 6. Auflage, Stuttgart/Berlin/Köln 2000, Rdnr. 87, 89, S. $35 \mathrm{f}$.

11 Zum föderalen System nach der deutschen Einheit vgl. Hartmut Klatt, Deutsche Einheit und bundesstaatliche Ordnung, in: VerwArch, 82 (1991), S. $430 \mathrm{ff}$.

12 Vgl. Edin Šarčević, Das Bundesstaatsprinzip, Tübingen 2000, S. 59 ff.; Karl-E. Hain, in: Christian Starck (Hrsg.), Kommentar zum Grundgesetz, Band 2, 5. Auflage, München 2005, Art. 79 Abs. 3, Rdnr. 131 f., S. 2251 f.

13 Vgl. Klaus Stern, Das Staatsrecht der Bundesrepublik Deutschland, Band II, München 1980, $\S 27$ I 1, S. 112. 
gegeben, so bezüglich der Paulskirchen-Verfassung von 1849, der Weimarer Verfassung von 1919 sowie des Grundgesetzes von 1949. ${ }^{14}$ Und auch im Verfassungskonvent von Herrenchiemsee wurde die Zusammensetzung des föderalen Organs so kontrovers diskutiert, dass der Chiemseer Entwurf schließlich beide Alternativen, sowohl das Bundesrats- als auch das Senatsmodell, enthielt. ${ }^{15}$ Für das Senatsmodell sprachen vor allem das Demokratieprinzip wegen der Wahl der Senatoren als Vertreter des Volkes auf Landesebene, die sich durch ihre Unabhängigkeit von der Parteibürokratie unterscheiden würden, eine damit einhergehende stärkere Kontrollfunktion gegenüber der Regierung sowie eine prognostizierte bessere Funktionsfähigkeit. Dagegen setzten sich die Argumente für das Bundesratsmodell mit seiner verfassungsgeschichtlichen Kontinuität letztendlich durch, wonach die mittelbar demokratisch legitimierte zweite Kammer die politischen Machtverhältnisse besser widerspiegeln sowie vom Sachverstand der Landesregierungen profitieren würde. ${ }^{16}$

Zwar wird das Bundesratsmodell nach herrschender Auffassung nicht von der Ewigkeitsgarantie des Art. 79 Abs. 3 GG erfasst, ${ }^{17}$ es dürfte aber trotz wiederholter Vorschläge für ein Senats- oder Mischmodell ${ }^{18}$ als fester Bestandteil staatsorganisatorischer Strukturen unumkehrbar geworden sein. ${ }^{19}$

\section{Mitgliedschaft}

Aus der Entscheidung für das Ratsprinzip im Grundgesetz folgt nach herrschender Auffassung ${ }^{20}$ und Rechtsprechung ${ }^{21}$ die Repräsentanz der Länder durch ihre Regierungen im Bundesrat und nicht wie nach dem Senatsprinzip die Repräsentanz der Völker der Gliedstaaten. Die dabei vorliegende Überlagerung des Demokratieprinzips durch das Bundesstaatsprinzip wird insbesondere bei der Sitz- und Stimmenverteilung deut-

14 Vgl. Heinz Laufer, Reform des Bundesrates?, in: Bundesrat (Hrsg.) (Fn. 7), S. 402 ff.; Angela Bauer-Kirsch, Herrenchiemsee. Der Verfassungskonvent von Herrenchiemsee - Wegbereiter des Parlamentarischen Rates, Bonn 2005, S. 100 f.; Julia Schmidt, Die Struktur der Zweiten Kammer im Rechtsvergleich. Ein Beitrag zur Reform des Bundesrates, Baden-Baden 2006, S. $72 \mathrm{ff}$.

15 Art. 66 und 67 Abs. 1 Chiemseer Entwurf.

16 Vgl. Konrad Reuter, Praxishandbuch Bundesrat, 2. Auflage, Heidelberg 2007, Art. 51 GG, Rdnr. 7 ff., S. $172 \mathrm{ff}$.

17 Vgl. Matthias Jestadt, in: Josef Isensee/Paul Kirchhof (Hrsg.) (Fn. 9), Rdnr. 48 ff., S. 821 ff.; Julia Schmidt (Fn. 14), S. 132 ff.

18 Für ein Senatsmodell vgl. Julia Schmidt (Fn. 14), S. 332 ff.; dies., Strukturelle Alternativen der Ausgestaltung des Bundesrates, in: DÖV, 59 (2006) 9, S. 379 ff. Vgl. hierzu auch Michael Sachs, Das parlamentarische Regierungssystem und der Bundesrat - Entwicklungsstand und Reformbedarf, in: VVDStRL, 58 (1999), S. 49 ff.

19 Vgl. Enquête-Kommission Verfassungsreform des Deutschen Bundestages, in: BT-Drs. 7/5924 vom 9.12.1976, S. 97 f.; Detlef Merten, Weiterer Reformbedarf im Bundesstaatsrecht, in: Wolfgang Durner/Franz-Joseph Peine (Hrsg.) (Fn. 5), S. 78.

20 Vgl. Klaus Stern, Das Staatsrecht der Bundesrepublik Deutschland, Band I, 2. Auflage, München 1984, § 19 III 8, S. 733; Hartmut Bauer, in: Horst Dreier (Hrsg.), Grundgesetz. Kommentar, Band II, 2. Auflage, Tübingen 2006, Art. 51, Rdnr. 12, S. 1254.

21 BVerfGE 8, 104 (120). 
lich. ${ }^{22}$ Eine Annäherung an das Demokratieprinzip kann gleichwohl durch eine angemessene Repräsentation der Länder nach bestimmten Kriterien, wie der Bevölkerungsgröße, erfolgen.

Der Bundesrat besteht aus „Mitgliedern der Regierungen der Länder“ (Art. 51 Abs. 1 Satz 1 GG). ${ }^{23}$ Gemäß dem Wortlaut dieser verfassungsrechtlichen Bestimmung müssten die weisungsgebundenen Mitglieder der Landesregierungen als Amtswalter auch die Mitglieder des Bundesrates sein, ${ }^{24}$ die ihrerseits die Bundesratssitze besetzen. ${ }^{25}$ Diese Interpretation des Art. 51 Abs. 1 Satz 1 GG wird durch die Rechtsprechung des Bundesverfassungsgerichts gestützt. ${ }^{26}$ Dabei folgt nach herrschender Auffassung in der Literatur ${ }^{27}$ und Rechtsprechung ${ }^{28}$ die Weisungsgebundenheit der Bundesratsmitglieder aus dem Fehlen einer der Weisungsungebundenheit der Abgeordneten des Bundestages vergleichbaren Bestimmung wie Art. 38 Abs. 1 GG sowie der einheitlichen Stimmabgabe der Länder im Bundesrat nach Art. 51 Abs. 3 Satz 2 GG. Außerdem lässt sich aus den Regelungen in Art. 77 Abs. 2 Satz 3 GG und Art. 53 a Abs. 1 Satz 3, 2. HS GG, wonach die Mitglieder des Gemeinsamen Ausschusses und des Vermittlungsausschusses nicht an Weisungen gebunden sind, schließen, dass sie Ausnahmenvorschriften zu der grundsätzlichen Weisungsgebundenheit der Bundesratsmitglieder sind. ${ }^{29}$

Nach anderer in der Literatur vertretener Auffassung sind die Länder Mitglieder des Bundesrates. ${ }^{30}$ Als Vertretungsorgan der Länder sei der Bundesrat selbst nicht handlungsfähig und müsse deshalb durch die Landesregierungen und diese durch die Landesminister im Bundesrat vertreten werden. ${ }^{31}$ Die insbesondere auf der systematischen Auslegungsmethode basierende Auffassung, wonach in Regelungen zur Beschlussfassung im Bundestag von „Mitgliedern“ des Bundestages (Mehrheit seiner Mitglie-

22 Vgl. Hartmut Bauer, in: Horst Dreier (Hrsg.) (Fn. 20), Art. 51, Rdnr. 21, S. 1258.

23 Vgl. Klaus Stern (Fn. 13), § 27 III 1, S. 134.

24 Zum Konzept der personalen Mitgliedschaft vgl. Herbert Küpper, Die Mitgliedschaft im Bundesrat, in: Der Staat, 42 (2003) 3, S. $391 \mathrm{ff}$.

25 Vgl. Jörn Ipsen, Staatsrecht I, 21. Auflage, Köln 2009, Rdnr. 340, S. 94; Gerhard Robbers, in: Michael Sachs (Hrsg.), Grundgesetz. Kommentar, 5. Auflage, München 2009, Art. 51, Rdnr. 1, S. 1281; Stefan Korioth, in: Christian Starck (Hrsg.) (Fn. 12), Art. 51 Abs. 1, Rdnr. 2, S. 1350; Bodo Pieroth, in: Hans Jarass/Bodo Pieroth, Grundgesetz für die Bundesrepublik Deutschland. Kommentar, 10. Auflage, München 2009, Art. 51, Rdnr. 1, S. 725; Hartmut Bauer, in: Horst Dreier (Hrsg.) (Fn. 20), Art. 51, Rdnr. 12, S. 1254.

26 BVerfGE 106, 310 (330).

27 Vgl. Gerhard Robbers, in: Michael Sachs (Hrsg.) (Fn. 25), Art. 51, Rdnr. 16, S. 1284; Horst Risse, in: Dieter Hömig (Hrsg.), Grundgesetz für die Bundesrepublik Deutschland, 8. Auflage, Baden-Baden 2007, Art. 51, Rdnr. 3, S. 398; Stefan Korioth, in: Christian Starck (Hrsg.) (Fn. 12), Art. 51, Rdnr. 23 ff., S. 1362 ff.; Bodo Pieroth, in: Hans Jarass/Bodo Pieroth (Fn. 25), Art. 51, Rdnr. 6, S. 681.

28 BVerfGE 8, 104 (120 f.).

29 Vgl. Stefan Korioth, in: Christian Starck (Hrsg.) (Fn. 12), Art. 51, Rdnr. 23, S. 1362; Gesine Wulfert-Markert, Stimmabgabe im Bundesrat - Von der Verfassung des Deutschen Reiches von 1871 bis zum Grundgesetz, Berlin 2005, S. 97 f.

30 Vgl. Konrad Reuter (Fn. 16), Art. 51 GG, Rdnr. 18, S. 180; Hartmut Maurer, Mitgliedschaft und Stimmrecht im Bundesrat, in: Hans-Detlef Horn (Hrsg.), FS für Walter Schmitt Glaeser, Berlin 2003, S. $157 \mathrm{ff}$.

31 So Hartmut Maurer (Fn. 30), S. 165. 
der), ${ }^{32}$ im Bundesrat dagegen von „Stimmen“ des Bundesrates (Mehrheit seiner Stimmen $)^{33}$ die Rede ist, verweist auf die Unterscheidung zwischen Mitgliedschaft und Stimmrecht. Während der Begriff „Stimme“ sich auf die Rechte der Länder in ihrer Mitwirkung bei der Willensbildung beziehe, beinhalte der Begriff „Mitglied“ die den Bundesratsmitgliedern ad personam zustehenden Rechte. ${ }^{34}$ Da es aber ein Stimmrecht ohne Mitgliedschaft nicht gebe, könne von einer Art Doppelmitgliedschaft: institutionelle Mitgliedschaft der Länder und persönliche Mitgliedschaft der Landesminister ausgegangen werden, ${ }^{35}$ womit sich der bestehende Auffassungsstreit, der für die Sitzverteilung von eher theoretischer Bedeutung ist, wieder relativiert. Letztendlich zählen im Bundesrat nicht die Mitglieder, sondern die Stimmen. ${ }^{36}$ Von praktischer Bedeutung sind die Fragen nach der Stimmenverteilung und der Stimmabgabe, denen noch nachzugehen sein wird.

\section{Sitzverteilung}

Für die Festsetzung der Anzahl der Sitze im föderalen Organ stehen zwei verschiedene Hauptmodelle zur Verfügung. Nach dem föderalen Prinzip der Gleichheit der Gliedstaaten würde diesen eine gleiche Anzahl von Sitzen zuerkannt werden oder nach ihrem „Gewicht" in räumlicher, demografischer, wirtschaftlicher oder politischer Hinsicht eine unterschiedliche Anzahl von Sitzen. ${ }^{37}$ Dabei wohnt der Zuerkennung einer unterschiedlichen Anzahl von Sitzen in Abhängigkeit von der Bevölkerungszahl ein demokratisches Element inne. Die Anzahl der Mitglieder, die jedes Land entsenden kann, richtet sich gemäß Art. 51 Abs. 3 Satz 1 GG nach der in Art. 51 Abs. 2 GG festgesetzten Anzahl der Stimmen. ${ }^{38}$

Würde die Sitzverteilung im Bundesrat ausschließlich auf der Grundlage des föderalen Prinzips erfolgen, müsste allen Bundesländern nach dem Prinzip der Gleichheit der Gliedstaaten die gleiche Anzahl von Sitzen zuerkannt werden. Diese Regel findet in föderalen Staaten wie den USA oder Australien Anwendung, die für das Senatsprinzip optiert haben. Zwar werden hier die Mitglieder des föderalen Organs unmittelbar durch das Volk gewählt und nicht wie nach dem Bundesstaatsprinzip von den Regierungen der Gliedstaaten bestellt, die Senatoren repräsentieren aber nicht eine verhältnismäßig gleiche Anzahl von Wählern. Das Senatsprinzip entspricht den demokratischen Grundsätzen der Volkssouveränität und der Repräsentation, es weist aber ein Demokratiedefizit hinsichtlich der Wahlrechtsgleichheit auf. Dagegen fließt in das im Grundgesetz gewählten Modell der Sitzverteilung im Bundesrat, in dem das föderale Prinzip und das Demokratieprinzip im Wege praktischer Konkordanz zu einem Ausgleich geführt werden sollen, die unterschiedliche Größe der Bevölkerung ein.

32 Art. 67 Abs. 1, 68 Abs. 1 Satz 2, 80 a Abs. 3 GG.

33 Art. 52 Abs. 3 Satz 1, 115 d Abs. 2 Satz 3 GG.

34 Vgl. Hartmut Maurer (Fn. 30), S. $160 \mathrm{f}$.

35 So ebenda, S. 165.

36 Vgl. Dr. Schmidt, in: Parlamentarischer Rat, Verhandlungen des Hauptausschusses, 40. Sitzung vom 14.1.1949, Bonn 1948/49, S. 508.

37 Vgl. Hartmut Bauer, in: Horst Dreier (Hrsg.) (Fn. 20), Art. 51, Rdnr. 3, S. 1249.

38 Vgl. Gerhard Robbers, in: Michael Sachs (Hrsg.) (Fn. 25), Art. 51, Rdnr. 13, S. 1283. 


\section{Stimmenverteilung im Bundesrat}

\section{Stimmengewichtung}

a) Formelle versus materielle Gleichheit der Bundesländer

Die Stimmenverteilung in einem föderalen Organ bestimmt den Einfluss der Gliedstaaten auf den Prozess der politischen Willensbildung des Bundes. Dabei besteht die Wahl zwischen zwei Alternativen: gleiche Anzahl von Stimmen nach der formellen Gleichheit $^{39}$ oder unterschiedliche Anzahl von Stimmen (Stimmengewichtung) in Abhängigkeit von bestimmten Kriterien nach der materiellen Gleichheit. Im Vergleich mit föderalen Organen anderer Staaten lässt sich bei der Stimmenverteilung eine Entwicklung hin zur materiellen Gleichheit beobachten. ${ }^{40}$

Mit Art. 51 Abs. 2 GG ist eine politische Entscheidung für die Stimmengewichtung verfassungsrechtlich verankert worden. ${ }^{41}$ Der in den Beziehungen der Bundesländer untereinander und in den Beziehungen des Bundes zu den Ländern geltende Grundsatz der Gleichbehandlung der Länder, der sich aus der Gleichheit der Länder als Staaten ergibt, gilt innerhalb des Bundesrates nicht als striktes Gebot. ${ }^{42}$ Daraus leitet sich eine Staffelung der Stimmen der Bundesländer ab. Die verfassungsrechtlich abgestufte Anzahl von Stimmen erkennt die Staatlichkeit der Gliedstaaten durch eine Mindeststimmenzahl (drei Stimmen) nach dem Gleichheitsprinzip an, nimmt dann aber eine Unterscheidung nach bestimmten Kriterien, hier demografischen, vor. ${ }^{43}$ In der dadurch angestrebten Repräsentanz sollen sich sowohl das Bundesstaatsprinzip als auch das Demokratieprinzip widerspiegeln.

Die deutsche Verfassungstradition fortsetzend, ${ }^{44}$ geht das Grundgesetz vom bundesstaatlichen Prinzip der Eigenstaatlichkeit der Gliedstaaten ${ }^{45}$ für die Bemessung der Stimmen der Länder im Bundesrat auf der Grundlage der Einwohnerzahlen als objektiven Maßstab aus. ${ }^{46}$ Indem der „demokratische Bundesstaat“ (Art. 20 Abs. 1 GG) die Anerkennung der Länder auch in ihrer Eigenstaatlichkeit und Vielfalt voraussetzt, können neben der Bevölkerungszahl auch andere Faktoren, wie historische Erfahrung, politische Zukunft, wirtschaftliche und soziale Entwicklung Berücksichtigung finden, die das Gewicht der Länder mitbestimmen.

39 So vorgeschlagen von Uwe Wagschal/Maximilian Grasl, Die modifizierte Senatslösung, in: ZParl, 35 (2004) 4, S. 747 f.

40 Vgl. Stefan Korioth, in: Christian Starck (Hrsg.) (Fn. 12), Art. 51 Abs. 2, Rdnr. 15, S. 1357.

41 Vgl. Jürgen Jekewitz, Die Stimmenverteilung im Bundesrat nach dem Einigungsvertrag, in: RuP, 27 (1991) 2, S. 98 ff.

42 Vgl. Konrad Reuter (Fn. 16), Art. 51 GG, Rdnr. 33, S. 187.

43 Stern spricht von einem, Mittelweg zwischen Gleichheit und Bevölkerungsarithmetik“. Klaus Stern (Fn. 13), § 27 III 3, S. 141.

44 Vgl. Kurt Behnke, Die Gleichheit der Länder im deutschen Bundesstaatsrecht, Berlin 1926, S. $70 \mathrm{ff}$.

45 BVerfGE 34, 216 (231).

46 Vgl. Roman Herzog, Zusammensetzung und Verfahren des Bundesrates, in: Isensee/Paul Kirchhof (Hrsg.), HdbStR, Band II, 2. Auflage, Heidelberg 1998, § 46, Rdnr. 1, S. 506. 
b) Stimmenstaffelung nach Einwohnerzahl

In Art. 51 Abs. 2 GG wird der Begriff des Einwohners und nicht wie in Art. 20 Abs. 2 der Begriff des (deutschen) Volkes verwendet. ${ }^{47}$ Nach dem allgemeinen Sprachgebrauch fallen unter den Einwohnerbegriff alle natürlichen Personen mit einem nicht nur vorübergehenden Wohnsitz im Landesgebiet unabhängig von ihrer Staatsangehörigkeit. ${ }^{48}$ Die Stimmenzahl eines Landes spiegelt eben nicht die gleiche demokratische Repräsentanz der Bürger wider, sondern den relativen Einfluss des Landes im Bundesrat. ${ }^{49}$ In die staatliche Gemeinwohlverpflichtung sind alle Einwohner einzubeziehen. Durch die von der Bevölkerungsproportionalität abweichende Stimmenstaffelung im Bundesrat gewichtet die Verfassung die staatsbürgerliche Gleichheit gering. ${ }^{50}$ Demzufolge ist die Einwohnerzahl als ein unbedenklicher Maßstab anzusehen. ${ }^{51}$ Aufgrund der Repräsentanz der Länder durch ihre Regierungen nach dem Ratsprinzip kann die Bezugnahme auf den Einwohnerbegriff als verfassungsgemäß gewertet werden. Ein Verstoß gegen den Grundsatz der Volkssouveränität ${ }^{52}$ ist auch nach herrschender Auffassung wegen der Überlagerung des Demokratieprinzips durch das Bundesstaatsprinzip nicht gegeben. ${ }^{53}$

Die Anzahl der Stimmen richtet sich gemäß der inhaltlichen Ausformungsbestimmung in $\S 27$ GOBR $^{54}$ nach den Ergebnissen der amtlichen Bevölkerungsfortschreibung, falls keine Ergebnisse einer amtlichen Volkszählung vorliegen. ${ }^{55}$ Diese Regelung wird von der in Art. 52 Abs. 3 Satz 2 GG geregelten Geschäftsordnungsautonomie des Bundesrates allerdings nicht erfasst. ${ }^{56}$ Das Grundgesetz regelt die Anzahl der Stimmen der Länder und damit die der Sitze gestaffelt in vier Stufen nach Einwohnerzahlen. Es fehlt aber eine Regelung des in Art. 51 Abs. 2 GG verwendeten Einwohnerbegriffes sowie eines Verfahrens zur Ermittlung der Einwohnerzahlen. Hierfür ist zumindest eine bundesgesetzliche Regelung erforderlich. ${ }^{57}$

47 BVerfGE 83, 37 (50). Vgl. Friedrich E. Schnapp, in: Ingo von Münch/Philip Kunig (Hrsg.), Grundgesetz. Kommentar, Band 2, 5. Auflage, München 2001, Art. 20, Rdnr. 20, S. 9; Michael Sachs, in: Michael Sachs (Hrsg.) (Fn. 25), Art. 20, Rdnr. 27 a, S. 782; Karl-Peter Sommermann, in: Christian Starck (Hrsg.) (Fn. 12), Art. 20, Rdnr. 148, S. 62 f.

48 Vgl. Carsten Deecke, Verfassungsrechtliche Anforderungen an die Stimmenverteilung im Bundesrat, Berlin 1998, S. 136; Walter Krebs, in: Ingo von Münch/Philip Kunig (Hrsg.) (Fn. 47), Art. 51, Rdnr. 12, S. 930.

49 Vgl. Michael Sachs (Fn. 18), S. 54.

50 Vgl. Konrad Reuter (Fn. 16), Art. 51 GG, Rdnr. 37, S. 192.

51 Vgl. Michael Sachs (Fn. 18), S. 54.

52 So Theodor Maunz/Rupert Scholz, in: Theodor Maunz/Günter Dürig (Hrsg.), Grundgesetz. Kommentar, Band IV, München 1996, Art. 51, Rdnr. 3, S. 2 ff.

53 So Walter Krebs, in: Ingo von Münch/Philip Kunig (Hrsg.) (Fn. 47), Art. 51, Rdnr. 12, S. 930; Hartmut Bauer, in: Horst Dreier (Hrsg.) (Fn. 20), Art. 51, Rdnr. 21, S. 1258 f.; Gerhard Robbers, in: Michael Sachs (Hrsg.) (Fn. 25), Art. 51, Rdnr. 12, S. 1283; Hartmut Maurer, Staatsrecht I, 5. Auflage, München 2007, Rdnr. 7, S. 490 f.

54 GOBR vom 26.11.1993 (BGB1. 1993 I, 2007; BGB1. 2007 I, 1057).

55 Vgl. Konrad Reuter (Fn. 16), Art. 51 GG, Rdnr. 37 ff., S. 190 ff., § 27 GO, Rdnr. 2 ff., S. 494 ff.

56 Vgl. Carsten Deecke (Fn. 48), S. 136; Hartmut Bauer, in: Horst Dreier (Hrsg.) (Fn. 20), Art. 51, Rdnr. 21, S. 1259.

57 So Carsten Deecke (Fn. 48), S. 136; Hartmut Bauer, in: Horst Dreier (Hrsg.) (Fn. 20), Art. 51, Rdnr. 21, S. 1259. 


\section{Veränderung der Stimmengewichtung}

Wie die Enquête-Kommission Verfassungsreform des Deutschen Bundestages 1976 bestätigt hat, ist eine bestimmte Staffelung der Stimmen der Länder staatstheoretisch nicht vorgegeben. ${ }^{58}$ Dafür, dass die Stimmenverteilung im Bundesrat ${ }^{59}$ nach der Regel der unterschiedlichen Anzahl von Stimmen der Bundesländer ${ }^{60}$ jedoch nicht einer arithmetisch exakten Repräsentation entspricht, ${ }^{61}$ hat sich der ihr zugrunde liegende Art. 51 Abs. 2 GG lange Zeit in einem „Dornröschenschlaf“ befunden. ${ }^{62}$ Die unlängst von Maurer vertretene, auf der These von der Ländermitgliedschaft im Bundesrat basierende Auffassung, wonach jedes Land nur eine Stimme hat, die mehrfach gezählt wird, ${ }^{63}$ ändert nichts an der unterschiedlichen Gewichtung der Stimmen der Länder, da hier den Stimmen ein unterschiedlicher Wert zugeordnet wird. Für die „quantitative Stimmendifferenzierung“, wie Stern sie bezeichnet, gebe es keine logische oder staatstheoretisch exakte Begründung, sie sei gleichwohl „maßvoll“ und „antihegemoniell“. Sie stelle einen „Mittelweg zwischen Gleichheit und Bevölkerungsarithmetik“"64 dar. ${ }^{65}$ Diese Staffelung erfuhr auch keine Modifikation bei den Änderungen der Stimmenverteilung von $1952^{66}$ und $1957 .{ }^{67}$

Dennoch war die Stimmenstaffelung im Bundesrat nicht unumstritten. Vor allem die Landesregierung von Nordrhein-Westfalen forderte wegen der großen Bevölkerungszahl ihres Landes ein größeres Stimmengewicht. ${ }^{68}$ Die daraufhin ins Leben gerufene Enquête-Kommission bestätigte aber in ihrem Schlussbericht vom 9. Dezember 1976 das bestehende Bundesratsmodell. ${ }^{69}$ Eine Neuregelung sei nur dann geboten, wenn es zu erheblichen Veränderungen in der Einwohnerzahl und der Grenzziehung gekommen wäre. Erst mit der deutschen Wiedervereinigung ${ }^{70}$ erfuhr die ursprüngliche Stimmenverteilung von drei bis fünf Stimmen ${ }^{71}$ eine Aufstockung um eine Stufe. ${ }^{72}$ Seither hat sich die Stimmenverteilung 1996 aufgrund der Überschreitung der in Art. 51 Abs. 2 GG festgesetzten Bevölkerungsgrenze durch Hessen verändert. Erstmals in der Geschichte

58 BT-Drs. 7/5924, S. 99 f.; Konrad Reuter (Fn. 16), Art. 51 GG, Rdnr. 34, S. 188.

59 Zur Zusammensetzung des Bundesrates von 1949 bis 2006 vgl. Konrad Reuter (Fn. 16), § 27 GO, Rdnr. 13, S. $500 \mathrm{ff}$.

60 So Pleyer, der von einer unterschiedlich großen Zahl an gleichwertigen Stimmen ausgeht. Vgl. Marcus C.F. Pleyer, Föderative Gleichheit, Berlin 2005, S. 161.

61 BT-Drs. 7/5924, S. 99 f. Vgl. Konrad Reuter (Fn. 16), Art. 51 GG, Rdnr. 33, S. 187.

62 Vgl. Carsten Deecke (Fn. 48), S. 17.

63 Hartmut Maurer (Fn. 30), S. 164.

64 Klaus Stern (Fn. 13), § 27 III 3, S. 141.

65 Vgl. Carsten Deecke (Fn. 48), S. 18 ff.

66 Aus Baden, Württemberg-Baden und Württemberg-Hohenzollern entstand das Bundesland Baden-Württemberg.

67 Beitritt des Saarlandes zum Geltungsbereich des Grundgesetzes.

68 Vgl. Peter Schindler, Der Bundesrat in parteipolitischer Auseinandersetzung, in: ZParl, 3 (1972), S. 149.

69 BT-Drs. 7/5924, S. 100.

70 Vgl. Eckart Busch, Die Stimmenverteilung im Bundesrat, in: ZG, 5 (1990) 4, S. 308 ff.

71 Vgl. Edgar Hein, Stimmenverteilung im Bundesrat, in: Die demokratische Gemeinde, (1972), S. 105.

$72 \mathrm{Zu}$ den Hintergründen der Verfassungsänderung von 1990 vgl. Stefan Korioth, in: Christian Starck (Hrsg.) (Fn. 12), Art. 51 Abs. 2, Rdnr. 18 f., S. 1359 f. 
des Grundgesetzes konnte sich die flexible Regelung zur Stimmenstaffelung in der Praxis bewähren. ${ }^{73}$

\section{a) Vorschlag Bayerns und Baden-Württembergs}

Infolge der deutschen Wiedervereinigung erarbeiteten Bayern mit Unterstützung Baden-Württembergs ${ }^{74}$ und Nordrhein-Westfalen ${ }^{75}$ Vorschläge für eine Änderung der Stimmenstaffelung nach Art. 51 Abs. 2 GG. Mit dem Beitritt der neuen Bundesländer sollte ein ausgewogenes Stimmenverhältnis zwischen kleinen, mittleren und großen Ländern weiterhin gewahrt bleiben. Es war beabsichtigt, die Stimmenrelation im Bundesrat stärker an die Relation der Einwohnerzahlen der Länder in einem geeinten Deutschland anzupassen. ${ }^{76}$ Damit die großen Länder nicht unverhältnismäßig an Stimmengewicht einbüßen und ihre Sperrminorität gegen Verfassungsänderungen verlieren, ist eine stärkere Spreizung der Stimmenverteilung nach der Bevölkerungszahl vorgeschlagen worden. ${ }^{77}$

Der Vorschlag Bayerns und Baden-Württembergs bezüglich einer Änderung des Art. 51 Abs. 2 GG sah eine unveränderte Mindestzahl von drei Stimmen je Land vor. Weiterhin sollten Länder mit zwei bis drei Millionen Einwohnern vier Stimmen erhalten, Länder mit drei bis fünf Millionen Einwohnern fünf Stimmen, Länder mit fünf bis sieben Millionen Einwohnern sechs Stimmen und Länder mit über sieben Millionen Einwohnern sieben Stimmen. Nach dieser um eine weitere Stufe erhöhten Stimmenstaffelung würden die großen Länder Nordrhein-Westfalen, Bayern, Baden-Württemberg und Niedersachsen je sieben Stimmen, Hessen sechs Stimmen, Sachsen, RheinladPfalz und Berlin je fünf Stimmen, Sachsen-Anhalt, Brandenburg, Schleswig-Holstein, Thüringen und Mecklenburg-Vorpommern je vier Stimmen sowie Hamburg, Saarland und Bremen je drei Stimmen erhalten. ${ }^{78}$ Durch die Zuerkennung einer zusätzlichen Stimme für die vier großen Länder (Nordrhein-Westfalen, Bayern, Baden-Württemberg und Niedersachsen) sowie Hessen, Sachsen, Rheinlad-Pfalz und Berlin würde sich die Anzahl der Stimmen im Bundesrat auf 78 erhöhen.

Lag der relative Einfluss (Stimmen/Bevölkerung) Nordrhein-Westfalens als bevölkerungsreichstes Bundesland mit bisher sechs Stimmen bei $0,33^{79}$ hätte er sich mit sieben Stimmen nur unwesentlich auf 0,39 erhöht. Hingegen wäre der relative Einfluss von Bremen als bevölkerungsschwächstes Land unverändert bei 4,5280 geblieben.

Mit diesem Vorschlag einer Neuregelung der Stimmengewichtung im Bundesrat sollten die vier größten Länder, die nach Art. 51 Abs. 2 GG sechs Stimmen innehaben, ihren stärkeren Einfluss im Bundesrat erhalten. Dies betrifft insbesondere die Sperrminorität von einem Drittel der Stimmen gegen Verfassungsänderungen gemäß Art. 79 Abs. 2

73 Vgl. Carsten Deecke (Fn. 48), S. 33 f.

74 BR-Drs. 551/90 vom 3.8.1990. Vgl. Hartmut Klatt (Fn. 11), S. 437.

75 BR-Drs. 557/90 vom 7.8.1990.

76 BR-Drs. 551/90, S. 7.

77 Ebenda, S. 1.

78 Ebenda, S. $1 \mathrm{ff}$.

79 Relativer Einfluss $=6$ Stimmen : 18,08 Mio. Einwohner.

80 Relativer Einfluss $=3$ Stimmen : 0,663 Mio. Einwohner. 
GG. ${ }^{81}$ Die vier Bundesländer repräsentieren ungefähr 49 Millionen der insgesamt ca. 82 Millionen Deutschen. Andererseits wird durch die geltende Begrenzung auf maximal sechs Stimmen pro Land eine Majorisierung durch ein oder zwei größere Länder im Bundesrat verhindert. ${ }^{82}$

\section{b) Vorschlag Nordrhein-Westfalens}

Der Vorschlag Nordrhein-Westfalens zur Änderung des Art. 51 Abs. 2 GG behielt die Mindestzahl von drei Stimmen pro Land ebenfalls bei. Sodann sollten Länder mit mehr als sechs Millionen Einwohnern fünf Stimmen, Länder mit mehr als sieben Millionen Einwohnern sechs Stimmen, Länder mit mehr als zehn Millionen Einwohnern sieben Stimmen und Länder mit mehr als fünfzehn Millionen Einwohnern acht Stimmen zugesprochen bekommen. ${ }^{83}$ Im Gegensatz zum Vorschlag Bayerns und Baden-Württembergs, wonach insgesamt acht Länder je eine zusätzliche Stimme erhalten hätten, wären es nach diesem Vorschlag lediglich zwei Länder (Nordrhein-Westfalen und Bayern), deren Stimmenzahl sich um zwei bzw. um eine erhöhen würde. Damit gäbe es nur eine geringfügige Erhöhung des relativen Einflusses dieser beiden Länder, Nordrhein-Westfalens von gegenwärtig 0,33 auf 0,44 und Bayerns von 0,48 auf $0,56 .{ }^{84}$ Die Gesamtstimmenzahl läge hiernach bei 72 .

Die um eine Stufe nach dem Vorschlag Bayerns und Baden-Württembergs bzw. um zwei Stufen nach dem Vorschlag Nordrhein-Westfalens erhöhte Stimmenstaffelung ergibt einen gleichen Durchschnittswert des Stimmenanteils. Beide Vorschläge haben einseitig die Stimmenstaffelung nach oben erweitert, nicht jedoch nach unten. Der Regelungsbedarf hätte eben nur in Bezug auf die großen Länder bestanden, d.h. so genannte „alte“ Bundesländer, nicht jedoch auf die kleinen und mittleren. ${ }^{85}$ Die nicht angenommenen Vorschläge hätten es demzufolge auch kaum vermocht, einen besseren Ausgleich als bisher zwischen der dem Bundesstaatsprinzip entsprechenden Gleichheit der Länder und der im Demokratieprinzip gründenden angemessenen Repräsentation der Landesbevölkerungen zu erreichen.

\section{c) Eigener Vorschlag}

Da die Stimmenverteilung im Bundesrat nicht auf einer strikten Proportionalität der Bevölkerungsgröße basiert, variiert das Verhältnis zwischen Anzahl der Stimmen und Bevölkerung bzw. der relative Einfluss der Bevölkerungen der Bundesländer nach den geltenden Verfassungsbestimmungen erheblich. Selbst wenn zusätzliche Kriterien (historische, politische, wirtschaftliche, soziale) für die bestehende Stimmenverteilung zugrunde gelegt würden, ist eine Überrepräsentation Bremens (relativer Einfluss: 4,52)

81 Vgl. Carsten Deecke (Fn. 48), S. 33.

82 Vgl. Heinz Laufer, Der Bundesrat, Bonn 1972, S. 10.

83 BR-Drs. 557/90, S. 3.

84 Relativer Einfluss $=6$ Stimmen : 12,42 Mio. Einwohner bzw. relativer Einfluss $=7$ Stimmen : 12,42 Mio. Einwohner.

85 BR-Drs. 557/90, S. 5 f. 
und eine entsprechende Unterrepräsentation Nordrhein-Westfalens (relativer Einfluss: $0,33)$ kaum zu rechtfertigen.

Nachfolgender eigener Vorschlag würde den Stimmenanteil der Länder im Bundesrat nach ihren Bevölkerungsanteilen entsprechend anpassen. Dabei wäre eine Staffelung der Bevölkerungsgrößen in mehrere Gruppen sowohl nach oben als auch nach unten zugrunde zu legen und eine weiter gestaffelte Anzahl von Stimmen diesen zuzuordnen. Der Vorschlag sieht für Länder mit bis zu drei Millionen Einwohnern zwei Stimmen, für Länder mit bis zu sechs Millionen Einwohnern drei Stimmen, für Länder mit bis zu neun Millionen Einwohnern vier Stimmen, für Länder mit bis zu zwölf Millionen Einwohnern fünf Stimmen, für Länder mit bis zu fünfzehn Millionen Einwohnern sechs Stimmen und für Länder mit mehr als fünfzehn Millionen Einwohner sieben Stimmen vor. Danach erhielten Nordrhein-Westfalen sieben Stimmen (+ 1 Stimme), Bayern sechs Stimmen (+/- 0 Stimmen), Baden-Württemberg fünf Stimmen (- 1 Stimme), Niedersachsen vier Stimmen (- 2 Stimmen), Hessen vier Stimmen (- 1 Stimme), Sachsen, Rheinland-Pfalz und Berlin je drei Stimmen (- 1 Stimme), Schleswig-Holstein, Brandenburg, Sachsen-Anhalt und Thüringen je zwei Stimmen (- 2 Stimmen), Hamburg, Mecklenburg-Vorpommern, Saarland und Bremen je zwei Stimmen (- 1 Stimme).

Nach diesem Vorschlag würden sechs anstelle von vier Gruppen nach Bevölkerungsgrößen gebildet und die Anzahl der Stimmen um jeweils eine verringert auf zwei bzw. erhöht auf sieben. Damit kann die Differenz des Verhältnisses zwischen Anzahl der Stimmen und Bevölkerung der Länder von bisher 4,1986 auf 2,62 ${ }^{87}$ reduziert werden. Die Gesamtstimmenzahl im Bundesrat würde von gegenwärtig 69 auf 51 sinken. Eine wesentliche Veränderung eines möglichen Abstimmungsergebnisses bei Anwendung der Abstimmungsregel der absoluten Mehrheit unter Einteilung der Länder in größere und kleinere ergibt sich jedoch nicht. Die Stimmen der kleineren Länder könnten die absolute Mehrheit erreichen, die der größeren nicht. ${ }^{88}$ Die Sperrminorität der größeren Länder gegen Verfassungsänderungen würde ebenfalls beibehalten. Das vorgeschlagene Modell könnte nachweislich einen besseren Ausgleich zwischen dem Bundesstaatsprinzip mit der Gleichheit der Länder und dem Demokratieprinzip mit der Repräsentation der Bevölkerung der Länder erreichen als das bisherige. Für eine Änderung der Stimmengewichtung in Art. 51 Abs. 2 GG bedürfte es allerdings einer Verfassungsänderung.

\section{Abschaffung der Stimmengewichtung}

Der Kompromiss zwischen der dem Bundesstaatsprinzip (Föderalismus) entsprechenden Gleichheit der Länder ${ }^{89}$ und der dem Demokratieprinzip entsprechenden angemessenen Repräsentanz der Bevölkerung der Länder (Volkssouveränität) ist durchaus nicht

86 Relativer Einfluss Bremens: 4,52 - relativer Einfluss Nordrhein-Westfalens: 0,33.

87 Relativer Einfluss Bremens: 3,01 - relativer Einfluss Nordrhein-Westfalens: 0,39.

88 Kleinere Länder nach Art. 51 Abs. 2 GG: Länder mit 3 und 4 Stimmen (insgesamt 40 Stimmen); nach eigenem Vorschlag: Länder mit 2, 3 und 4 Stimmen (insgesamt 32 Stimmen). Größere Länder nach Art. 51 Abs. 2 GG: Länder mit 5 und 6 Stimmen (insgesamt 29 Stimmen); nach eigenem Vorschlag: Länder mit 5, 6 und 7 Stimmen (insgesamt 18 Stimmen).

89 Vgl. Hans Kelsen, Allgemeine Staatslehre, Berlin 1925, S. 217 f. 
zwingend. So regelt das Grundgesetz in einigen Bestimmungen eine Gleichbehandlung der Länder in Bundesorganen (Art. 53 a Abs. 1 Satz 3, Art. 95 Abs. 2 GG). ${ }^{90}$

Bei einer Abschaffung der Stimmengewichtung würde jedes Bundesland die gleiche Anzahl von Stimmen im Bundesrat zugesprochen bekommen unabhängig von der Einwohnerzahl oder sonstigen Kriterien. Die auf dem Prinzip der formellen Gleichheit der Länder basierende Stimmenverteilung unterstreicht die Einzelstaatlichkeit im Rahmen des Föderalismus und bestimmt damit den gleichen Einfluss jedes Landes, ob groß oder klein, auf die Willensbildung und Entscheidungsfindung im föderalen Organ.

Das Modell „Ein Staat - eine Stimme“ entspricht auf völkerrechtlicher Ebene der Stimmenverteilung in Organen internationaler Organisationen, wie z.B. der UN-Generalversammlung, wo nach dem Prinzip der souveränen Gleichheit (Art. 2 Ziff. 1 UNCharta) jedes Mitglied gem. Art. 18 Abs. 1 UN-Charta eine Stimme unabhängig von der Größe der Bevölkerung, des Territoriums, der wirtschaftlichen oder politischen Kraft oder sonstigen Kriterien hat.

Auch die EU schafft stufenweise die Stimmengewichtung im Rat bei Entscheidungsfindungen mit qualifizierter Mehrheit (Art. 205 Abs. 2 EU a.F.) ab. Weil ein entsprechender Ausgleich zwischen dem völkerrechtlichen Prinzip der souveränen Gleichheit der Mitgliedstaaten und der dem Demokratieprinzip entsprechenden angemessenen Repräsentanz der Bevölkerung der Mitgliedstaaten im Rat kaum erreicht werden kann, sieht der seit dem 1. Dezember 2009 geltende Reformvertrag von Lissabon (Art. 16 Abs. 4 EU n.F. i.V.m. Art. 238 Abs. 2 AEU) vor, die Stimmengewichtung im Rat aufzuheben und durch eine neue Definition der qualifizierten Mehrheit zu ersetzen, die allerdings durch die geforderte Mehrheit der Bevölkerung ebenfalls eine demografische Mehrheit impliziert. ${ }^{91}$

Wenn auch die Unterschiede zwischen den Gliedstaaten im Bundesrat der Bundesrepublik Deutschland nicht so groß ausfallen wie beispielsweise auf der EU- oder gar UN-Ebene, so sind sie dennoch vorhanden und dies nicht nur in Bezug auf die Größe der Bevölkerung, sondern eben auch in wirtschaftlicher und finanzieller Hinsicht. Ausdruck dessen ist der Länderfinanzausgleich, mit dem zum Zwecke eines angemessenen Ausgleiches der Finanzkraftunterschiede unter den Ländern Ausgleichszahlungen reicherer Bundesländer (Geberländer) an ärmere Bundesländer (Nehmerländer) vorgenommen werden. ${ }^{92}$ Die Übernahme der Stimmenverteilungsregel „Ein Staat - eine Stimme“ im Bundesrat würde vor allem aber ein Vorherrschen des Bundesstaatsprinzips und ein Ausblenden des Demokratieprinzips aufgrund der Nichtbeachtung der Landeseinwohner zur Folge haben. Damit sollte die Regel der gleichen Stimmenzahl für die Gliedstaaten keine weiter zu verfolgende Alternative für das Bundesratsmodell darstellen.

90 Vgl. Michael Sachs (Fn. 18), S. 53.

91 Vgl. Volker Epping, in: Christoph Vedder/Wolff Heintschel von Heinegg (Hrsg.), Europäischer Verfassungsvertrag, Baden-Baden 2007, Art. I-25, Rdnr. 2, S. 136.

92 Finanzausgleichsgesetz vom 20.12.2001 (BGBl. 2001 I, 3955, 3956), zuletzt geändert durch Artikel 11 des Gesetzes vom 22.12.2009 (BGB1. 2009 I, 3950). 


\section{Stimmabgabe im Bundesrat}

\section{Gebot der einheitlichen Stimmabgabe}

Das Modell der Stimmenverteilung im Bundesrat nach demografischen Kriterien schließt ein, dass die zugeteilten Stimmen eines Landes nur einheitlich und nur durch anwesende Mitglieder oder deren Vertreter abgegeben werden können (Art. 51 Abs. 3 Satz 2 GG). ${ }^{93}$ Damit bestimmt das Grundgesetz die Einheitlichkeit der Stimmabgabe eines Landes. ${ }^{94}$ Dies fließt aus dem imperativen Mandat der Mitglieder des Bundesrates. ${ }^{95}$ Die verfassungsrechtlichen Bestimmungen regeln demzufolge nicht nur wie viele Stimmen den Mitgliedern des kollegialen Entscheidungsorgans zustehen, sondern auch wie diese abzugeben sind. Den Ländern steht zwar ein unterschiedliches Stimmengewicht in Abhängigkeit von der Einwohnerzahl zu, sie können aber nur mit einem Willen im Bundesrat vertreten sein. ${ }^{96}$ Damit die Stimmabgabe im Bundesrat einheitlich erfolgt, legen die Verfassungen einiger Länder eine vorherige Beschlussfassung der Landesregierung zwecks Stimmabgabe im Bundesrat fest. ${ }^{97}$

Eine uneinheitliche Stimmabgabe trat erstmalig am 19. Dezember 1949 auf der 10. Sitzung des Bundesrates auf, als das Bundesland Nordrhein-Westfalen durch zwei seiner Landesminister bei der Abstimmung über eine Anordnung uneinheitlich votiert hatte. ${ }^{98}$ Diese „Panne“ konnte durch eine Klarstellung des Ministerpräsidenten behoben werden. In der Lehre fehlte eine Einigung über die Folgen einer widersprüchlichen Stimmabgabe. Nach Ansicht einiger Autoren komme es maßgeblich auf die Stimme des Ministerpräsidenten bzw. Kabinettsvorsitzenden an, ${ }^{99}$ nach der herrschenden Meinung sind alle uneinheitlich abgegebenen Stimmen des Landes ungültig. ${ }^{100}$

Zum zweiten Mal in der Geschichte der deutschen Verfassungspraxis hat sich das theoretische Problem der uneinheitlichen Stimmabgabe auf der 774. Sitzung des Bun-

93 Vgl. Reinhold Zippelius/Thomas Würtenberger, Deutsches Staatsrecht, 32. Auflage, München 2008, Rdnr. 10, S. 416; Heinrich de Wall, in: Karl Heinrich Friauf/Wolfram Höfling (Hrsg.), Berliner Kommentar zum Grundgesetz, Band 2, Berlin 2006, Art. 51, Rdnr. 26, S. 12; Konrad Reuter (Fn. 16), Art. 51 GG, Rdnr. 44 ff., S. 196 ff.

94 BVerfGE 106, 310 (331).

95 Vgl. Klaus Stern (Fn. 13), § 27 III 2, S. 136; Herbert Küpper (Fn. 24), S. 397; Stefan Korioth, in: Christian Starck (Hrsg.) (Fn. 12), Art. 51 Abs. 3, Rdnr. 23, S. 1362.

96 Vgl. Hans Schäfer, Der Bundesrat, Köln/Berlin 1955, S. 51; Gesine Wulfert-Markert (Fn. 29), S. 105.

97 Art. 49 Abs. 2 LV BW, Art. 37 Abs. 2 Ziff. 2 LV NI, Art. 64 Abs. 1 LV SN, Art. 68 Abs. 3 Ziff. 2 LV ST, Art. 76 Abs. 2 Satz 1 LV TH.

98 Vgl. Roland Fritz/Karl-Heinz Hohm, „Szenen einer Abstimmung“, in: AuAS, 11 (2002), S. 15.

99 Vgl. Klaus Stern (Fn. 13), § 27 III 2, S. 136 f.; Dieter Blumenwitz, in: Rudolf Dolzer/Klaus Vogel (Hrsg.), Bonner Kommentar zum Grundgesetz, Band 6, Heidelberg (Stand 1987), Art. 51, Rdnr. 29, S. 24.

100 Vgl. Wolf-Rüdiger Schenke, Die verfassungswidrige Bundesratsabstimmung, in: NJW, 56 (2002) 18, S. 1320 f.; Hartmut Maurer (Fn. 30), S. 177; Gerhard Robbers, in: Michael Sachs (Hrsg.) (Fn. 25), Art. 51, Rdnr. 15, S. 1283 f.; Stefan Korioth, in: Christian Starck (Hrsg.) (Fn. 12), Art. 51 Abs. 3, Rdnr. 21, S. 1361; Bodo Pieroth, in: Hans Jarass/Bodo Pieroth (Fn. 25), Art. 51, Rdnr. 6, S. 726. 
desrates am 22. März 2002, ${ }^{101}$ auf der zwei Landesminister und Bundesratsmitglieder des Landes Brandenburg uneinheitlich über das Zuwanderungsgesetz abstimmten, in ein praktisches und prozessuales transponiert. ${ }^{102}$ Die Rechtsauffassungen zu dieser Frage polarisieren sich einerseits auf ein eigenständiges Stimmrecht jedes Bundesratsmitgliedes ${ }^{103}$ und andererseits auf die „Stimmführerschaft“ des Ministerpräsidenten ${ }^{104}$ mit unterschiedlichen Rechtsfolgen auch innerhalb dieser Auffassungen. In seiner Entscheidung tritt das Bundesverfassungsgericht der Auslegung entgegen, wonach der Art. 51 Abs. 3 Satz 2 GG die Bundesratsmitglieder des Landes rechtlich zu einer einheitlichen Stimmabgabe verpflichte. ${ }^{105}$ Die Bestimmung verbiete es lediglich, einen gespaltenen Landeswillen im Abstimmungsergebnis des Bundesrates durch Aufteilung der Stimmen des Landes zu berücksichtigen. Eine uneinheitliche Stimmabgabe sei also ungültig, aber nicht verfassungswidrig. ${ }^{106}$

Der Rechtsstreit über das Abstimmungsergebnis zum Zuwanderungsgesetz ist, wie Schmitz zutreffend formuliert, ein anschauliches Beispiel dafür, wie ,[...] farblose Verfahrensvorschriften des Staatsorganisationsrechts zu interessanten Fällen führen können, die eine breite Öffentlichkeit ansprechen." "107

101 Stenographischer Bericht BR (Fn. 1), S. 131 ff., Abstimmung S. $171 \mathrm{ff}$.

102 Vgl. Kerstin Odendahl, Das Erfordernis der einheitlichen Stimmabgabe im Bundesrat, in: JuS, 42 (2002) 11, S. 1051 ff.; Joachim Lang, Das Urteil des Bundesverfassungsgerichts zum Zuwanderungsgesetz, in: ZParl, 34 (2003) 3, S. 596 ff.

103 Vgl. Wolf-Rüdiger Schenke (Fn. 100), S. 1318 ff.; Claus-Peter Bienert, Zur Frage unterschiedlichen Abstimmungsverhaltens der Mitglieder der Regierung eines Bundeslandes bei der Abstimmung im Bundesrat, in: ThürVBl., 11 (2002) 5, S. 108 ff.; Florian Becker, Die uneinheitliche Stimmabgabe im Bundesrat, in: NVwZ, 21 (2002) 5, S. 569 ff.; Jörn Ipsen, Gespaltenes Votum bei Abstimmungen im Bundesrat, in: DVB1. 117 (2002) 10, S. 653 ff.; Rolf Gröschner, Das Zuwanderungsgesetz im Bundesrat, in: JZ, 57 (2002) 13, S. 621 ff.; Thilo Tetzlaff, Die Bedeutung des Landesverfassungsrechts bei der Beurteilung der Abstimmung über das Zuwanderungsgesetz im Bundesrat, in; DÖV, 56 (2003) 17, S. 693 ff.

104 Vgl. Carsten F. Soerensen, Die verfassungsgemäße Bundesratsabstimmung zum Zuwanderungsgesetz, in: NJW, 55 (2002) 24, S. XII ff.; Roland Fritz/Karl-Heinz Hohm (Fn. 98), S. 11 ff.; Dieter Dörr/Heinrich Wilms, Verfassungsmäßigkeit der Abstimmung über das Zuwanderungsgesetz?, in: ZRP, 35 (2002) 6, S. 265 ff.; Jürgen Jekewitz, Der Streit über das Zuwanderungsgesetz, in: RuP, 38 (2002) 1, S. 83 ff.; Hans Meyer, Abstimmungsstreit im Bundesrat, in: Hans Meyer (Hrsg.), Abstimmungskonflikt im Bundesrat im Spiegel der Staatsrechtslehre, Baden-Baden 2003, S. 146 ff.; Gerrit Mulert (Fn. 3), S. 29.

105 Eine Pflicht zur einheitlichen Stimmabgabe schlägt Jekewitz vor. Vgl. Jürgen Jekewitz, Deutscher Föderalismus, in: RuP, 40 (2004) 2, S. 97.

106 BVerfGE 106, 310 (335). Vgl. Stefan Korioth, in: Christian Starck (Hrsg.) (Fn. 12), Art. 51 Abs. 3, Rdnr. 21, S. 1361.

107 Thomas Schmitz, Constitutional Jurisprudence Federal Republic of Germany, in: ERPL/ REDP, 15 (2003) 4, S. $1428 \mathrm{ff}$. 


\section{Abschaffung der einheitlichen Stimmabgabe}

Eine Änderung diesbezüglicher Verfahrensvorschriften ist in der Literatur ${ }^{108}$ und auch im Zusammenhang mit der Föderalismusreform in der 15. Legislaturperiode durch die Bundesstaatskommission von Bundestag und Bundesrat zur Modernisierung der bundesstaatlichen Ordnung ${ }^{109}$ angeregt worden. Durch eine Abschaffung der Pflicht zur einheitlichen Stimmabgabe eines Bundeslandes könne der parteipolitischen Instrumentalisierung des Bundesrates entgegengewirkt und eine Umgehung des Mitwirkungsgebotes verhindert werden. ${ }^{110}$ Das Prinzip der einheitlichen Stimmabgabe sei unter Legitimationsgesichtspunkten ohnehin nur schwer vermittelbar. ${ }^{111}$

Die Bundesratsmitglieder haben bei den Abstimmungen im Bundesrat nicht ihren persönlichen Standpunkt zu vertreten, sondern denjenigen ihrer Landesregierung. Diese Verpflichtung entspringt dem Status der Mitglieder des Bundesrates als bestellte Mitglieder der Regierungen ihrer Länder. Eine Verpflichtung der Mitglieder des Bundesrates an Abstimmungen teilzunehmen und ihre Stimme abzugeben, ist jedoch weder im Grundgesetz noch in der Geschäftsordnung des Bundesrates vorgesehen. Eine allgemein verpflichtende Regelung zur Mitwirkung an der Entscheidungsfindung und damit zur Stimmabgabe lässt sich auch auf Länderebene nicht nachweisen. ${ }^{112}$ Dafür können die Landesregierungen durch Weisung ihre Mitglieder im Bundesrat in konkreten Fällen verpflichten, persönlich an der Abstimmung teilzunehmen und ihre Stimmabgabe vorzunehmen. ${ }^{113}$

Eine Änderung der Legitimationsgrundlage würde aber folgerichtig eine Änderung der Struktur des Bundesrates in Richtung einer parlamentarischen zweiten Kammer mit einer Aufhebung der Weisungsgebundenheit der Bundesratsmitglieder ${ }^{114}$ nach sich ziehen. Außerdem müsste eine Regel für die Sitzverteilung in den einzelnen Ländern festgelegt werden, die das Gewicht der Koalitionsparteien in den Landesregierungen widerspiegele. Damit können zwar die parteipolitischen Mehrheitsverhältnisse der Länder im Bundesrat besser abgebildet werden, kleinere Koalitionsparteien würden hingegen in eine Minderheitsposition gedrängt werden. Selbst wenn diese sich ohnehin schon in einer Minderheitsposition befinden, entspreche diese Regel jedenfalls nicht dem Bundesratsmodell, das die Gebietskörperschaft Land und nicht die Wählerschaft repräsentiere. Eine Rechtfertigung der Abschaffung der Pflicht zur einheitlichen Stimmabgabe

108 Vgl. Roland Sturm, Vorbilder für eine Bundesratsreform?, in: ZParl, 33 (2002) 1, S. 177; Frank Decker, Konturen des ,,neuen“ Föderalismus aus Expertensicht: Eine Zwischenbilanz der Arbeit der Kommission zur Modernisierung der bundesstaatlichen Ordnung, in: ZParl, 35 (2004) 3, S. 554; Peter M. Huber, Deutschland in der Föderalismusfalle?, Heidelberg 2003, S. 34; ders., Klarere Verantwortungsteilung von Bund, Ländern und Kommunen?, in: NJW, 58 (2004) 27, Beilage, S. 26.

109 Das Föderalismusreform-Begleitgesetz vom 5.9.2006 (BGBl. 2006 I, 2098) enthält keine Reformen zur Stimmabgabe oder Stimmenverteilung.

110 Vgl. Peter M. Huber, Klarere Verantwortungsteilung von Bund, Ländern und Kommunen? (Fn. 108), S. 26.

111 Vgl. Frank Decker (Fn. 108), S. 554.

112 Vgl. Jan Roscheck, Enthaltung und Nichtbeteiligung bei staatlichen Wahlen und Abstimmungen, Berlin 2003, S. 148.

113 Vgl. Konrad Reuter (Fn. 16), Art. 51, Rdnr. 81, S. 212.

114 Vgl. Stefan Korioth, in: Christian Starck (Hrsg.) (Fn. 12), Art. 51 Abs. 3, Rdnr. 21, S. 1361. 
und damit der Einführung des freien Mandats auf der Grundlage des Demokratieprinzips wäre nur dann gegeben, wenn die Bundesratsmitglieder von den Landesparlamenten oder direkt von den Wählern gewählt würden. ${ }^{115}$ Da dies nicht der Fall und eine nach außen einheitliche Vertretung der Länder durch ihre Regierungen nach dem Bundesstaatsprinzip geboten ist, ${ }^{116}$ sollte dem Vorschlag für eine Abschaffung der einheitlichen Stimmabgabe nicht nachgekommen werden.

\section{Einführung einer Stimmführerschaft}

In der Staatspraxis hat sich die Abstimmung durch einen Stimmführer herausgebildet und als zweckmäßig erwiesen. ${ }^{117}$ Dabei sind weder das Auftreten des Ministerpräsidenten als Stimmführer seines Landes ${ }^{118}$ noch die Einrichtung des Stimmführers ${ }^{119}$ überhaupt zwingend. „Das Grundgesetz erwartet die einheitliche Stimmabgabe und respektiert die Praxis der landesautonom bestimmten Stimmführer, ohne seinerseits mit Geboten und Festlegungen in den Verfassungsraum des Landes überzugreifen. "120 Aus dieser Konzeption des Grundgesetzes für den Bundesrat folge nach Ansicht des Bundesverfassungsgerichts, dass der Abgabe der Stimmen durch einen Stimmführer jederzeit durch ein anderes Bundesratsmitglied desselben Landes widersprochen werden kann und damit die Voraussetzungen der Stimmführerschaft insgesamt entfallen. ${ }^{121}$ Die durch das Grundgesetz anerkannte „Praxis der landesautonom bestimmten Stimmführer" sei nur solange verfassungskonform, wie kein anderes Bundesratsmitglied desselben Landes der abgegebenen Stimme durch den Stimmführer widerspreche. ${ }^{122}$ Eine Rückfrage an den Ministerpräsidenten könne auch nicht damit gerechtfertigt werden, dass dieser nach der Landesverfassung die Richtlinien der Politik bestimmt und demzufolge gegenüber den anderen Bundesratsmitgliedern seines Landes eine herausgehobene Stellung innehat. Rangverhältnisse des Landesverfassungsrechts spielen auf der Bundesebene keine Rolle. Damit erteilt das Bundesverfassungsgericht in Übereinstimmung mit der herrschenden Meinung der Ansicht, ${ }^{123}$ im Zweifel sei auf den Ministerpräsidenten als ,geborenen Stimmführer“ abzustellen, eine Absage. ${ }^{124}$

Die Einführung einer Stimmführerschaft würde bedeuten, dass bei uneinheitlicher Stimmabgabe der Bundesratsmitglieder desselben Landes die Stimme des Stimmführers ausschlaggebend wäre und eben nicht die Ungültigkeit aller Stimmen des betroffenen Landes bewirken würde. Somit stellt sich die Frage, ob die Stimmführerschaft rechtlich

115 Bundesstaatskommission von Bundestag und Bundesrat zur Modernisierung der bundesstaatlichen Ordnung, Arthur Benz, Abstimmungsverfahren im Bundesrat, Kommissionsdrucksache 0086 vom 28.10.2004, S. 2 f. Vgl. Gerrit Mulert, Die Funktion zweiter Kammern in Bundesstaaten, Baden-Baden 2006, S. $281 \mathrm{f}$.

116 Vgl. Jörn Ipsen (Fn. 103), S. 655; Julia Schmidt (Fn. 14), S. 296.

117 Vgl. Jörn Ipsen (Fn. 103), S. 655.

118 Vgl. ebenda.

119 Vgl. Konrad Reuter (Fn. 16), Art. 51 GG, Rdnr. 51, S. 197.

120 BVerfGE 106, 310 (330).

121 Ebenda (330 f.).

122 Ebenda.

123 Vgl. Klaus Stern (Fn. 13), § 27 III 2, S. 137.

124 BVerfGE 106, 310 (334). 
geregelt werden sollte. ${ }^{125}$ Eine rechtliche Regelung müsste sodann sowohl im Innenverhältnis in den Landesregierungen als auch im Außenverhältnis im Bundesrat erfolgen. Nach den Landesverfassungen einiger Länder ${ }^{126}$ bzw. der Praxis der übrigen beschließt die Landesregierung über die Stimmabgabe im Bundesrat. ${ }^{127}$ Mit diesem rechtsverbindlichen Beschluss wird im Innenverhältnis festgelegt, wie im Außenverhältnis im Bundesrat - abgestimmt werden soll. Allerdings fehlt bislang eine verbindliche Regelung darüber, wer im Bundesrat für das Land abstimmen darf. Dies könnte generell der Ministerpräsident oder speziell ein Mitglied der Landesregierung entsprechend seiner Ressortzuständigkeit in Abhängigkeit von der im Bundesrat abzustimmenden Sachentscheidung sein. Damit die notwendige landesinterne Regelung über die Stimmführerschaft auch im Außenverhältnis rechtswirksam wird, bedarf es einer weiteren Regelung zumindest in der Geschäftsordnung des Bundesrates, die in Form einer Erklärung der Landesregierung gegenüber dem Bundesratspräsidenten analog der Bestimmung des $\S 1$ GOBR ergehen könnte. Danach teilen die Landesregierungen dem Präsidenten des Bundesrates ihre Mitglieder des Bundesrates mit. ${ }^{128}$ Eine rechtswirksame Regelung der Stimmführerschaft würde die in Art. 51 Abs. 3 Satz 2 GG geforderte einheitliche Stimmabgabe garantieren. ${ }^{129}$ Zwar wäre dann eine Abgabe abweichender Stimmen immer noch möglich, aber diese würde nicht mehr zur Ungültigkeit aller Stimmen des Landes führen.

\section{Stimmenthaltung im Bundesrat}

Die Stimmenthaltung als rechtliches Institut der Beschlussfassung im Bundesrat bedeutet, dass ein stimmberechtigtes Mitglied weder positiv noch negativ auf das Zustandekommen des Beschlusses einzuwirken gedenkt. Mit der Stimmenthaltung sollte dem abstimmenden Bundesratsmitglied die Möglichkeit gegeben werden, in Bezug auf den Sachantrag neutral zu sein. ${ }^{130}$

Aus der Abstimmungsregel der absoluten Mehrheit in Art. 52 Abs. 3 Satz 1 GG folgt nun, dass Stimmenthaltungen im Bundesrat in der Regel eine destruktive Wirkung haben. Bei der Abstimmung über Einspruchsgesetze und bei Anrufung des Vermittlungsausschusses fehlen bei entsprechenden Stimmenthaltungen die Ja-Stimmen. Bei der Abstimmung über Zustimmungsgesetze kommen Stimmenthaltungen Nein-Stimmen gleich ( 330 Abs. 1 GOBR). Zur Vermeidung eben dieser negativen Folgen gibt es verschiedene rechtspolitische und verfassungsrechtliche Ansätze.

125 Vgl. Hartmut Maurer (Fn. 30), S. 174.

126 Art. 49 Abs. 2 LV BW, Art. 37 Abs. 2 Ziff. 2 LV NI, Art. 64 Abs. 1 LV SN, Art. 68 Abs. 3 Ziff. 2 LV ST, Art. 76 Abs. 2 Satz 1 LV TH; Konrad Reuter (Fn. 16), Art. 51 GG, Rdnr. 61, S. 203.

127 Vgl. Hartmut Maurer (Fn. 30), S. 173.

128 Vgl. ebenda, S. $174 \mathrm{f}$.

129 Vgl. ebenda, S. 176.

130 Vgl. Christoph Lambrecht, Die Stimmenthaltung bei Abstimmungen und die Nein-Stimme bei Wahlen, Frankfurt am Main 1988, S. 116. 


\section{Mainzer Bundesratsklausel}

Zunehmend sehen rechtlich nicht verbindliche Koalitionsvereinbarungen zwischen meist großen und kleinen Parteien der Länder über das Stimmverhalten im Bundesrat vor, dass im Falle eines Dissenses unter den Koalitionspartnern das Land sich seiner Stimmen im Bundesrat enthält. Mit diesem in der Regel Zugeständnis an den kleineren Koalitionspartner wird das Abstimmungsverhalten des Landes von einer Regierungsminderheit bestimmt, das mit dem Demokratieprinzip, das nach Art. 28 Abs. 1 GG auch als Strukturprinzip für die Länder gilt, nur schwerlich in Einklang zu bringen ist. Ein Verbot entsprechender politischer Koalitionsvereinbarungen ist aber schon wegen des Schutzes der Interessen kleinerer Koalitionspartner nicht sinnvoll. ${ }^{131}$

Zur Vermeidung einer Stimmenthaltung im Bundesrat ist in Rheinland-Pfalz ein Modell unter Bezugnahme auf das Losverfahren in die Koalitionsvereinbarung vom 30. April 1996 zwischen SPD und FDP für die 13. Wahlperiode als Novum eingeführt worden. Nach der so genannten Mainzer Bundesratsklausel entscheidet das Los nicht über das Abstimmungsverhalten, sondern legt fest, welcher der Koalitionspartner die so genannte Stimmführerschaft im Bundesrat bezüglich des ersten Tagesordnungspunktes im Bundesrat innehat. Zu wessen Gunsten das Los fällt, der darf die Stimme des Landes in seinem Interesse abgeben. Für die nächsten streitigen Tagesordnungspunkte findet das Losverfahren keine Anwendung mehr. Hier steht die Stimmführerschaft dem anderen Koalitionspartner alternierend zu. Dieses Verfahren wird bei Vorliegen mehrerer Dissenspunkte bis zum Ende durchgeführt. ${ }^{132}$ Dabei sind die Koalitionspartner nachdrücklich bemüht, strittige Sachfragen solange zu verhandeln, bis eine Kompromisslösung gefunden ist, so dass das auch hier angewendete Losverfahren zu einer ultima ratio wird.

Die zunächst vielfach verspottete Mainzer Bundesratsklausel hat durchaus Verfassungsgeschichte geschrieben. ${ }^{133}$ Wenn sich in einer realen politischen Situation bezüglich einer bestimmten Sachfrage die Koalitionspartner mit gleichwertigen Argumenten gegenüberstehen, sind in der Regel zwei Ausgänge möglich. Zum einen kann die Unmöglichkeit, die Patt-Situation aufzulösen, zur Stimmenthaltung oder Handlungsunfähigkeit führen. Bei möglicher Herstellung eines politischen Kompromisses würde zum anderen eine Entscheidung auf dem kleinsten gemeinsamen Nenner gefunden werden können, die meist nicht zukunftsweisend ist. Zur Herbeiführung einer sachgerechten, zukunftsweisenden, politischen Entscheidung bietet sich hier das Losverfahren an, mit dessen Hilfe eine von beiden Entscheidungen ausgewählt wird. So führt das Mainzer Losverfahren bei Sachentscheidungen zu einer klaren Entscheidung bezüglich der Stimmabgabe des Landes im Bundesrat und verhindert damit eine Stimmenthaltung, so dass eine Mitwirkung des Landes an der Sachentscheidung im Bundesrat gewährleistet

131 Bundesstaatskommission von Bundestag und Bundesrat zur Modernisierung der bundesstaatlichen Ordnung, Hans Meyer, Überlegungen zur Abstimmungsregel des Art. 52 Abs. 3 Satz 1 GG für den Bundesrat, in: Kommissionsdrucksache 0026 vom 3.3.2004, S. 3.

132 Vgl. Rüdiger Zuck, Politische Sekundärtugenden (III), in: NJW, 50 (1997) 5, S. 298.

133 Vgl. Siegfried Jutzi, Losentscheid im Gesetzgebungsverfahren, in: ZPR, 29 (1996) 10, S. $380 \mathrm{ff}$. 
wird. ${ }^{134}$ Da beide in der Regierungskoalition des Landes vertretenen Parteien aus demokratischen Wahlen hervorgegangen sind und die Interessen der Wähler repräsentieren, kommt es zu einer im Rahmen der freiheitlich-demokratischen Ordnung möglichen Entscheidung. Die in einer Koalition zusammengeschlossenen Regierungsparteien stimmen ohnehin in den wichtigsten politischen Zielen überein. Und da in einem demokratischen System jede politische Partei die Chance hat, Regierungspartei zu werden, dürfte es auch keine unvertretbare Sachentscheidung geben, und wenn doch, ist diese spätestens bei den nächsten Wahlen korrigierbar. ${ }^{135}$ Eine Vereinbarkeit der Mainzer Bundesratsklausel mit dem Demokratieprinzip ist somit zu bejahen. Die Mainzer Bundesratsklausel als politisches Instrument erfüllt den Zweck, Stimmenthaltungen im Bundesrat zu vermeiden. Verfassungsrechtlich lässt sie sich als unbedenklich bewerten. Die Kompetenz zur Entscheidung über die wechselnde Stimmführerschaft obliegt der Landesregierung, die verfassungsrechtlich gegenüber ihren Bundesratsmitgliedern weisungsbefugt ist. ${ }^{136}$

\section{Einführung eines Stimmenthaltungsverbots}

Zur Vermeidung einer Stimmenthaltung der Länder bei der Abstimmung über Zustimmungsgesetze ist im Rahmen der Föderalismusreform in der Bundesrepublik Deutschland in der 15. Legislaturperiode der Vorschlag eingebracht worden, einen neuen Satz 2 in Art. 53 Abs. 3 GG mit folgendem Wortlaut einzuführen: „Bei Abstimmungen über Gesetze, die der Zustimmung des Bundesrats bedürfen, sind Stimmenthaltungen nicht zulässig. " ${ }^{137}$ Da der Bundesrat aus Vertretern der Landesregierungen bestehe, die im Kollektiv für ihr Land abstimmen, haben diese auch Verantwortung über Entscheidungen zu übernehmen und können nicht, wie individuelle und weisungsungebundene Abgeordnete Stimmenthaltung üben. In der Regel praktizieren die Landesregierungen Stimmenthaltungen aus Rücksicht auf den Bestand der Regierungskoalition im Bund. Länderinteressen würden mit diesem Verhalten gerade nicht verfolgt. Der Verzicht auf Eigenständigkeit durch die Landesregierungen und das Nichtnachkommen ihrer Verantwortung gegenüber der Wählerschaft würden ein Stimmenthaltungsverbot begründen. ${ }^{138}$ Mit dem Scheitern der Bestrebungen einer Föderalismusreform in der 15. Legislaturperiode ist dieser Vorschlag nicht weiter ernsthaft verfolgt worden.

Mit einer Einführung eines Stimmenthaltungsverbots würde den Bundesratsmitgliedern eine mögliche Form der Teilnahme an der Abstimmung als Äußerung ihres Stimmrechts genommen werden. Da die Stimmenthaltung gerade bei der Anwendung der absoluten Mehrheitsregel negative Folgen bewirkt, wäre eine Änderung der Abstimmungsregel, bei der diese Folgen neutralisiert würden, vorzuziehen. ${ }^{139}$

134 Vgl. Alfred Rührmair, Der Bundesrat zwischen Verfassungsauftrag, Politik und Länderinteressen, Berlin 2001, S. 67.

135 Vgl. Rüdiger Zuck (Fn. 132), S. 299.

136 Vgl. Stefan Korioth, in: Christian Starck (Hrsg.) (Fn. 12), Art. 51 Abs. 3, Rdnr. 24, S. 1362 f.; Hartmut Bauer, in: Horst Dreier (Hrsg.) (Fn. 20), Art. 51, Rdnr. 27, S. 1263.

137 Arthur Benz (Fn. 115), S. 4.

138 Ebenda.

139 Vgl. Frank Decker (Fn. 108), S. 553. 


\section{Abstimmungsregel im Bundesrat}

\section{Absolute Mehrheit}

Für Abstimmungen im Bundesrat gilt nach Art. 52 Abs. 3 Satz 1 GG generell das Prinzip der Mehrheit seiner Stimmen, d.h. die absolute Mehrheit. ${ }^{140}$ Damit folgt das geltende Verfassungsrecht jedoch nicht der deutschen Verfassungstradition. Nach der Frankfurter Reichsverfassung war für das Staatenhaus (§98 Abs. 1), nach der Verfassung des Norddeutschen Bundes (Art. 7 Abs. 2 Satz 2) und der Bismarckschen Reichsverfassung (Art. 7 Abs. 3) für die Bundesräte und nach der Weimarer Verfassung für den Reichsrat (Art. 66 Abs. 4) einfache Stimmenmehrheit allgemein ausreichend.

Weil anfangs die geltende verfassungsrechtliche Bestimmung als Regelung der Beschlussfähigkeit interpretiert wurde, sind selbst mit einfacher Mehrheit votierte Anträge bis zur 36. Sitzung am 6. Oktober 1950 als wirksam zustande gekommene Beschlüsse angesehen worden. ${ }^{141}$ Danach hat die Vorschrift in $\S 13$ Abs. 2 GOBR vom 31. Juli 1953 die absolute Mehrheit für Beschlüsse des Bundesrates verlangt. ${ }^{142}$ In der geltenden Geschäftsordnung bestimmt $\S 30$ Abs. 1, ob ein Beschluss mit der Mehrheit seiner Stimmen, d.h. absoluten Mehrheit angenommen wurde oder nicht. ${ }^{143}$

Sowohl die absolute als auch die Zweidrittelmehrheit berechnen sich nach der Gesamtzahl der Stimmen der Bundesländer gemäß Art. 51 Abs. 2 GG. Bei dem insgesamt 69 Stimmen zählenden Bundesrat sind für die absolute Mehrheit 35 Stimmen erforderlich. Stimmenthaltungen und Nichtteilnahme an der Abstimmung werden letztendlich wie Nein-Stimmen gewertet. ${ }^{144}$ Infolgedessen wird eine Mehrheitsbildung erschwert.

\section{2. Änderung der Mehrheitsregel in einfache Mehrheit}

Die negativen Wirkungen der Stimmenthaltungen im Bundesrat könnten ausgeschlossen werden, indem die Abstimmungsregel in Art. 52 Abs. 3 Satz 1 GG von der absoluten in die einfache Mehrheit geändert werden würde. Danach wäre es ausreichend, wenn die positiven die negativen Stimmen überwiegen. Bei Erteilung der Zustimmung würde es zur Beschlussfassung kommen. Dieser Vorschlag geht maßgeblich auf eine Empfehlung der Bertelsmann-Stiftung ${ }^{145}$ zurück, die auch von der Bundesstaatskommission von Bundestag und Bundesrat zur Modernisierung der bundesstaatlichen Ordnung aufgegriffen wurde. ${ }^{146}$ Allerdings spricht der Vorschlag der Bertelsmann-Stiftung irrtüm-

140 BVerfGE 8, 274 (297). Stefan Korioth, in: Christian Starck (Hrsg.) (Fn. 12), Art. 52 Abs. 3, Rdnr. 12, S. 1372; Bodo Pieroth, in: Hans Jarass/Bodo Pieroth (Fn. 25), Art. 52, Rdnr. 6, S. 728; Konrad Reuter (Fn. 16), Art. 52 GG, Rdnr. 20, S. 238.

141 Vgl. Stenographischer Bericht BR, 36. Sitzung vom 6.10.1950, S. 633.

142 Vgl. Konrad Reuter (Fn. 16), Art. 52 GG, Rdnr. 19, S. 238.

143 Vgl. ebenda, § 30 GOBR, Rdnr. 2, S. 513.

144 Vgl. Hans Hofmann, in: Bruno Schmidt-Bleibtreu/Hans Hofmann/Axel Hopfauf (Hrsg.), Kommentar zum Grundgesetz, 11. Auflage, München 2008, Art. 52, Rdnr. 9, S. 1165 f.

145 Bertelsmann-Kommission „Verfassungspolitik \& Regierungsfähigkeit“ (Hrsg.), Entflechtung 2005: Zehn Vorschläge zur Optimierung der Regierungsfähigkeit im deutschen Föderalismus, Gütersloh 2000, S. 30 f.

146 Vgl. Hans Meyer (Fn. 131), S. 4. 
lich von der Umwandlung der absoluten in die relative Mehrheit, statt in die einfache Mehrheit. Unter der einfachen Mehrheit wird generell die Mehrheit, d.h. mehr als die Hälfte der abgegebenen Stimmen verstanden (Abstimmungsmehrheit). Danach kommt ein Beschluss zustande, wenn die Anzahl der Ja-Stimmen die der Nein-Stimmen um eine übersteigt, wobei Stimmenthaltungen nicht mitgezählt werden.

Gegen die Zustimmung mit einfacher Mehrheit wurden als Nachteile angeführt, dass „[...] ein Veto des Bundesrats nur von einer Minderheit der Länder gestützt wird“, ,[...] Koalitionsregierungen mit der Enthaltung taktische Entscheidungen treffen [können], ohne dafür sichtbar Verantwortung zu übernehmen“ und dass ,[...] die Stimmen von Ländern, die nicht dem Regierungs- oder Oppositionslager angehören an Gewicht zu verlieren [drohen]“. ${ }^{147}$ Diese aufgeführten Nachteile sind allerdings auch bei Anwendung der absoluten Mehrheitsregel nicht zu vermeiden.

Da in der gegenwärtigen politischen Parteien- und Regierungslandschaft Parteien, die nicht dem Regierungs- bzw. Oppositionslager angehören, ohnehin in einer Minderheit sind, werden ihre Stimmen auch keinen maßgeblichen Einfluss auf die Beschlussfassung im Bundesrat ausüben können. Eine Stimmenthaltung muss nicht zwangsläufig mit Nichtübernahme von Verantwortung gleichgesetzt werden. Die Anwesenheit und Teilnahme an der Abstimmung mindestens eines stimmberechtigten Vertreters der Länder vorausgesetzt, die alle Stimmen ihrer respektiven Länder zusammen abgeben können, ${ }^{148}$ werden zur Beschlussfassung im Bundesrat von insgesamt 69 Stimmen für die einfache Mehrheit genauso viele Stimmen (35) benötigt wie für die absolute Mehrheit. Bei Anwendung der einfachen Mehrheit kommen Stimmenthaltungen jedoch im Gegensatz zur absoluten Mehrheit keine Bedeutung zu. Da die Beschlussfähigkeitsregelung für den Bundesrat gemäß $§ 28$ Abs. 1 GOBR auf die Mehrheit seiner (gesetzlichen bzw. verfassungsrechtlichen) Stimmen abstellt, ist eine Beschlussfassung durch eine Minderzahl von Stimmen dennoch nicht zu befürchten.

Auch wäre mit einer Änderung der Mehrheitsregel ein Verlust an demokratischer Repräsentation oder Legitimität der Entscheidungen im Bundesrat nicht zwangsläufig gegeben. Die in der Geschäftsordnung des Bundesrates geregelte Beschlussfähigkeit von der Mehrheit seiner Stimmen, die der absoluten Mehrheit als Abstimmungsregel in Art. 52 Abs. 3 Satz 2 GG entspricht, garantiert bereits diese hinreichend.

Ein Übergang zur einfachen Mehrheitsregel (Abstimmungsmehrheit statt Mitgliedermehrheit), wie im Regelfall im Bundestag, würde zweifellos zu einer erhöhten Funktionsfähigkeit des Bundesrates beitragen. ${ }^{149}$ Dies bedürfte jedoch einer entsprechenden Verfassungsänderung.

147 Arthur Benz (Fn. 115), S. 2.

148 Vgl. Hans Hofmann, in: Bruno Schmidt-Bleibtreu/Hans Hofmann/Axel Hopfauf (Hrsg.) (Fn. 144), Art. 51, Rdnr. 17, S. 1157 f.

149 So Michael Sachs (Fn. 18), S. 76; implizit auch Herbert Küpper (Fn. 24), S. 408. Von relativer Mehrheit sprechen Hartmut Maurer (Fn. 30), S. 178; Roland Sturm (Fn. 108), S. 175 f.; ders., Zur Reform des Bundesrates, in: APuZ, B 29-30 (2003), S. 28; Uwe Wagschal/Maximilian Grasl (Fn. 39), S. 743; Gerrit Mulert (Fn. 115), S. 280. 


\section{Schlussbemerkung}

Nicht alle untersuchten Vorschläge zur Reform des Bundesrates entsprechen verfassungsrechtlichen Prinzipien oder tragen zur Verbesserung bestehender und kritisierter Funktions- und Effizienzdefizite dieses Verfassungsorgans bei.

Die verfassungsrechtliche Entscheidung für das Bundesratsmodell mit seiner verfassungsgeschichtlichen Kontinuität basiert auf der Repräsentanz der Länder durch ihre Regierungen im Bundesrat. Dieses Modell ist ungeachtet wiederholter Vorschläge für ein Senats- oder Mischmodell solider Bestandteil staatsorganisatorischer Strukturen. Das im Grundgesetz gewählte Modell der Sitzverteilung für den Bundesrat, in dem das föderale Prinzip und das Demokratieprinzip im Wege praktischer Konkordanz zu einem Ausgleich geführt werden sollen, berücksichtigt die unterschiedliche Größe der Landesbevölkerungen. Eine Regelung des in Art. 51 Abs. 2 GG verwendeten Einwohnerbegriffes sowie eines Verfahrens zur Ermittlung der Einwohnerzahlen fehlt aber bislang.

Die verfassungsrechtliche Stimmenstaffelung im Bundesrat gem. Art. 51 Abs. 2 GG ist reformbedürftig. Hier könnte eine erweiterte Stimmenstaffelung jeweils um eine zusätzliche Gruppe sowohl nach unten als auch nach oben erfolgen. Das vorgeschlagene Modell könnte einen besseren Ausgleich zwischen dem Bundesstaatsprinzip mit der Gleichheit der Länder und dem Demokratieprinzip mit der Repräsentation der Bevölkerung der Länder bewirken.

Dem Vorschlag, die Stimmengewichtung im Bundesrat abzuschaffen und durch die Stimmenverteilungsregel „Ein Staat - eine Stimme“ zu ersetzen, sollte indes nicht gefolgt werden. Dies würde vor allem ein Vorherrschen des Bundesstaatsprinzips und ein Ausblenden des Demokratieprinzips aufgrund der Nichtbeachtung der Landeseinwohner zur Folge haben.

Wegen des Gebots einer nach außen einheitlichen Vertretung der Länder durch ihre Regierungen nach dem Bundesstaatsprinzip sollte dem Vorschlag von einer Abschaffung der einheitlichen Stimmabgabe ebenfalls nicht nachgegangen werden. Um die in Art. 51 Abs. 3 Satz 2 GG geforderte einheitliche Stimmabgabe der Mitglieder desselben Landes zu garantieren, könnte die bereits gewohnheitsrechtlich bestehende Stimmführerschaft in der Geschäftsordnung des Bundesrates festgeschrieben werden.

Der Vorschlag von der Einführung eines Stimmenthaltungsverbots steht im Widerspruch zum Stimmrecht der Bundesratsmitglieder. Da die Stimmenthaltung gerade bei Anwendung der absoluten Mehrheitsregel negative Folgen bewirkt, sollte vielmehr die absolute Mehrheit als Abstimmungsregel durch die einfache Mehrheit ersetzt werden. Mit diesem Vorschlag, der einer Verfassungsänderung bedürfte, würde zur deutschen Verfassungstradition zurückgekehrt werden. 